\title{
La formación ciudadana en estudiantes de bachillerato
}

\author{
Jesús García Reyes
}

\section{Resumen}

En este trabajo se plantea un panorama de los esfuerzos realizados para incrementar la participación de los jóvenes de bachillerato mediante los proyectos curriculares de formación ciudadana en las escuelas y las iniciativas de los propios jóvenes. El objetivo de este artículo es distinguir la formación ciudadana de la cultura ciudadana, la primera como un elemento importante para la construcción de individuos dentro de las escuelas y la segunda como la participación y acción perenne en la vida cotidiana.

Palabras clave: ciudadanía, jóvenes, formación, bachillerato.

\section{Citizen education in high SCHOOl students}

\begin{abstract}
This paper presents an overview of the efforts made to increase the participation of young people in high school through the curricular projects of citizen training in schools, as well as the initiatives of the young people themselves. The aim of this article is to distinguish citizen formation from citizen culture, the first as an important element for the construction of individuals within the schools, and the second as a participation and a perennial action in daily life. We analyze some current positions on the training of citizens in high school and their involvement in the formation of citizens, in order to encourage the participation of young people in their daily lives to strengthen citizen culture.
\end{abstract}

Key words: cityzenship, teenagers, education, high school.

DOI: http://doi.org/10.22201/codeic.16076079e.2018.v19n5.a7 Recepción: 6/3/2017. Aprobación: 9/8/18. 
Jesús García Reyes

jgarcia8207@gmail.com

Lic. en Sociología por la FCPyS, UNAM; Mtro. en Pedagogía por la FFyL, UNAM y Dr. en Pedagogía por la FFyL, UNAM. Investigador de tiempo completo titular C.

\section{Introducción}

La formación ciudadana consiste en el desarrollo de aptitudes, destrezas, comportamientos y habilidades que hagan posible el respeto hacia el otro, para integrarlo y así llegar a acuerdos y consensos (Martínez et. al, 2010). El tema de la ciudadanía es fundamental en educación, porque es parte de los planes y programas de estudio en la educación media superior. En 2008 se estableció el Sistema Nacional de Bachillerato, en el cual se implementó el proyecto de reformar integralmente a la educación media superior en México, con la consigna de ampliar un marco de diversidad en donde estudiantes, maestros, padres de familia, autoridades e instituciones son partícipes (Secretaría de Educación Pública [SEP], 2008).

La Reforma de Instituciones de Educación Media Superior sugiere cambios al sistema educativo, entre ellos establecer el Servicio Profesional Docente como mecanismo de capacitación, formación y evaluación de maestros, directores, supervisores y de instalaciones. Además, se creó el Sistema Nacional de Evaluación, el cual aporta los lineamientos y directrices para el desarrollo de fortalezas y superación de debilidades en los maestros; asimismo, se lleva a cabo la evaluación de los planes y programas de estudio, de maestros, de directores y supervisores y de las instalaciones de la SEP (SEP, 2008). 


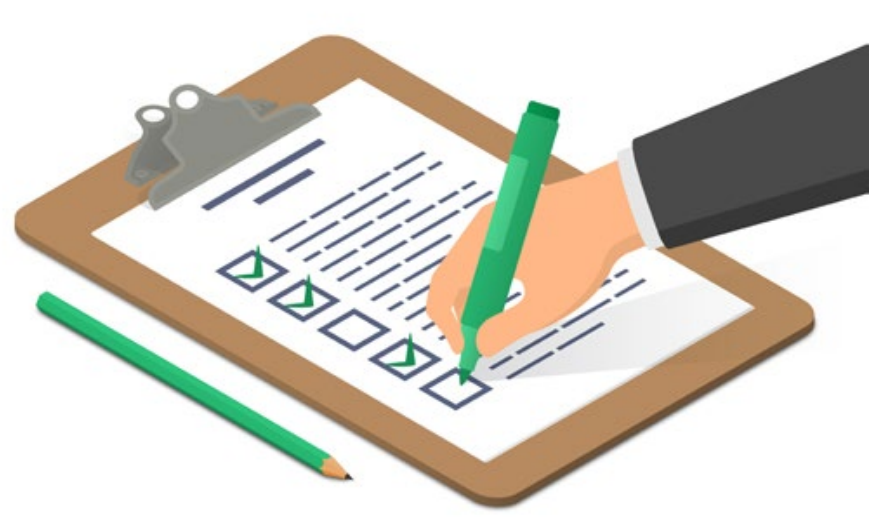

Sin embargo, los resultados no han sido favorables porque no se cuenta con la preparación suficiente, ni tampoco con la actualización a los docentes que les permita un dominio de los contenidos y una capacidad profesional para el enfoque de competencias (Alcántara y Zorrilla, 2010). Los efectos han sido contundentes con respecto al desempeño escolar por parte de los estudiantes. El estudio Panorama Educativo en Educación Media Superior (Instituto Nacional para la Evaluación de la Educación [INEE], 2015) señaló que los estudiantes de bachillerato no cuentan con la preparación suficiente para el campo profesional y, por lo tanto, para cursar estudios de nivel superior, ya que 15 de cada 100 alumnos no aprueban las materias. Aunado a la tasa de deserción escolar de 16.5\%, anualmente el desarrollo de los jóvenes para enfrentar la competencia laboral se encuentra comprometido. Con respecto a materias y temas de destreza y habilidades sociales, como es la formación ciudadana, los resultados son negativos, porque expresan una baja toma de decisiones acertadas ante situaciones donde se busca el bien común.

En general, el desempeño de los mexicanos se encuentra por debajo del promedio de la Organización para la Cooperación y el Desarrollo Económicos (OCDE) en temas de ciencias (416 puntos), lectura (423 puntos) y matemáticas (408 puntos). En estas tres áreas, sólo 1\% de los estudiantes en México logra alcanzar niveles de competencia de excelente con respecto a otros países de la Organización (OCDE, 2016).

Por ello se han implementado esfuerzos para lograr cambios en el mejoramiento de los planes y programas de estudio. De modo que, la SEP ha exhortado a las diversas instituciones de educación media a la actualización y modificación de sus contenidos, enfatizando el análisis y reflexión sobre la formación ciudadana de forma transversal durante el bachillerato. Se busca una perspectiva que sitúe al estudiante en un contexto que le permita comprender y valorar su entorno (SEP, 2012). De tal manera, las competencias adquiridas durante los cursos procuran que los estudiantes puedan distinguir los derechos y responsabilidades inherentes al ejercicio de la ciudadanía, a la promoción de la vida democrática, a partir de una dimensión multicultural y en la toma de decisiones responsables y comprometidas con su contexto (SEP, 2012).

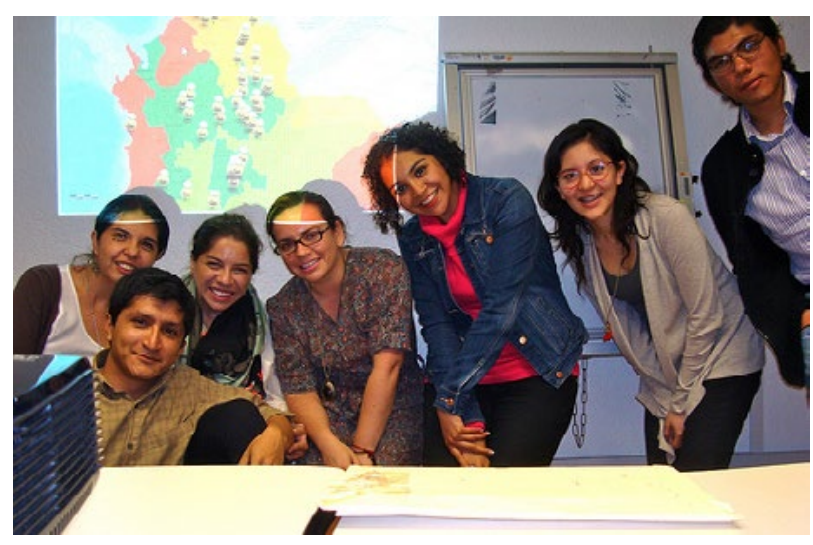


En el caso de los bachilleratos de la Universidad Nacional Autónoma de México (UNAM), se diseñaron planes diferenciados entre la Escuela Nacional Preparatoria (ENP) y el Colegio de Ciencias y Humanidades (CCH), para poder definir el perfil de ingreso, y actualizar los programas de estudio, de acuerdo con la esencia de cada una de las instituciones; así como formar y actualizar a los profesores para que incorporen estrategias para la enseñanza y el aprendizaje de los estudiantes (UNAM, 2012).

De ahí la necesidad de buscar alternativas para la formación ciudadana, porque solamente ceñirse a una propuesta curricular donde se prefijan en algunas asignaturas los contenidos actitudinales expresados en valores puede provocar la memorización de alguna idea, y en la mayoría de los casos estos contenidos quedan obsoletos o poco practicados por los profesores y estudiantes (Robles, 2013). Por tal motivo, surgieron propuestas para los planes de estudio acerca de la ciudadanía y su conformación en la vida cotidiana.

\section{Algunas propuestas para la formación ciudadana}

La formación de jóvenes ciudadanos en el cch busca, a partir de la inclusión de temas relacionados con la ciudadanía en las asignaturas Derecho I y ॥, el fomento de valores de colaboración, solidaridad y honestidad como parte integral en la formación de los alumnos para el mejoramiento de la vida social, un mayor compromiso y consciencia para participar en los temas políticos y sociales del país (Robles, 2013). La construcción de la ciudadanía mediante las relaciones cotidianas, a través del respeto y el trabajo en equipo dentro de las aulas, es de importancia en la práctica de los valores y promoción de los derechos humanos mediante el conocimiento de la ley y las obligaciones como ciudadanos.

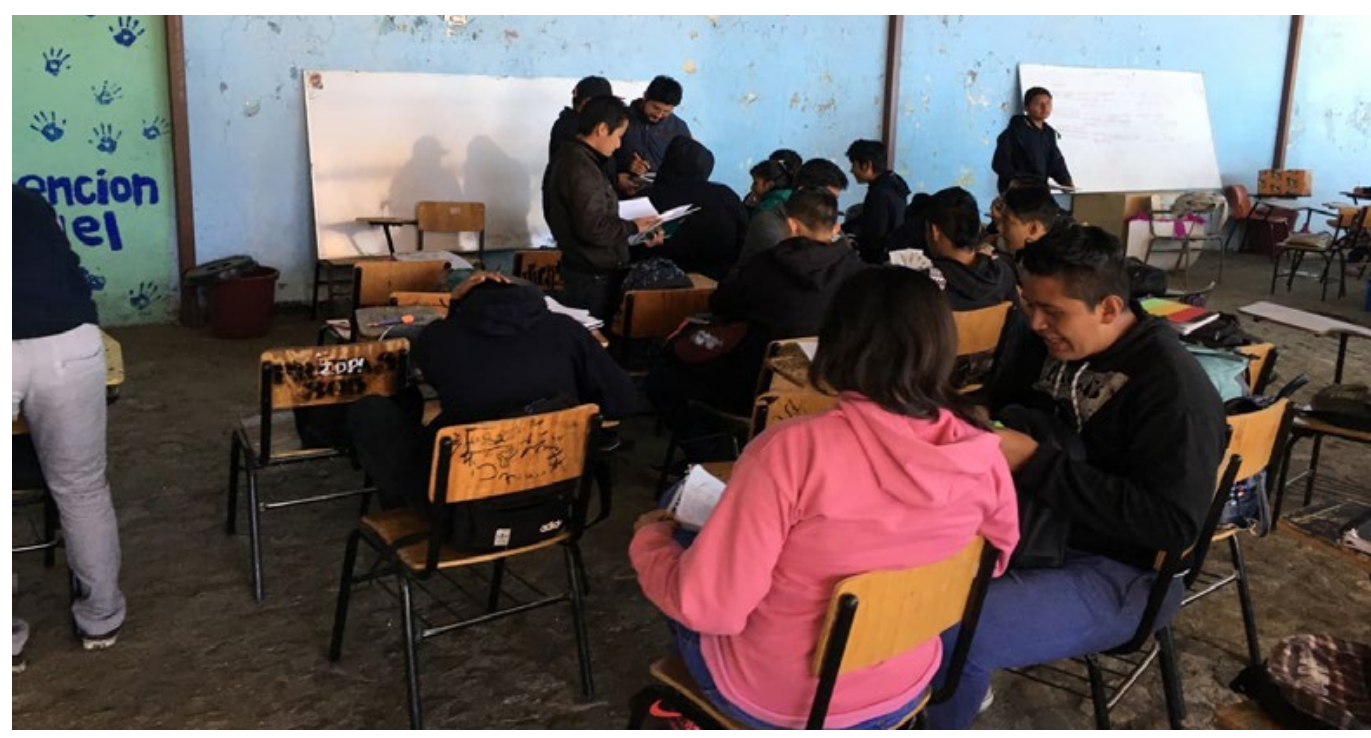




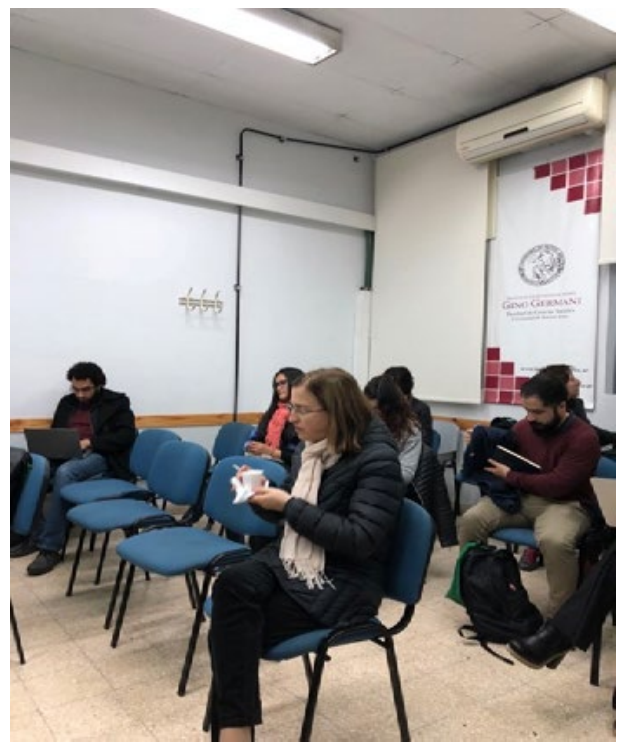

En la investigación La construcción de ciudadanía en la educación media superior: un estudio de caso sobre docentes de la UNAM, Castro et al. (2014) sostienen que la situación de los estudiantes como sujetos proclives a la formación de la ciudadanía desde el espacio escolar, la importancia de que los docentes sean capacitados y el propiciar espacios para la discusión, debate, acompañamiento, colaboración y solidaridad entre iguales, permite una mayor inclusión entre pares y vitaliza la cultura ciudadana en diversos contextos. Dentro del estudio, 33\% de los docentes del bachillerato muestra un interés acerca de los temas políticos; $62.5 \%$, un interés regular; y solamente $4.5 \%$ es apático hacia la política, lo que demuestra que hacen falta incentivos para ampliar la formación ciudadana y generar espacios propensos para la participación (Castro et al., 2014).

Bolívar y Belaguer (2014) proponen una revisión de los programas pedagógicos de la enseñanza de la educación ciudadana, para incentivar la formación de los estudiantes, a través del desarrollo de competencias críticas, destrezas y habilidades en la toma de decisiones cívicas, mediante el compromiso, la responsabilidad y la participación colectiva. Se plantea un escenario donde la educación juega un papel muy importante, para valorar la integración de la diversidad y donde no se discrimine. La educación ciudadana desde la pedagogía no sólo enseña valores, sino, también, la apertura de relaciones para el diálogo, debate y toma de decisiones. Se busca conciliar el pluralismo político con la creciente realidad multicultural a través del reconocimiento, el compromiso y la participación en el tejido social. Páges (2003) examina a la enseñanza de la historia en el bachillerato como un medio de investigación-acción, en el cual existe una relación entre una ciudadanía democrática y conciencia histórica, mediante un énfasis en la identidad nacional, la pertenencia cultural, y el reconocimiento al régimen sobre los derechos y obligaciones.

Otra propuesta la elabora Carrillo (2012), quien considera que las ciencias sociales y las humanidades son disciplinas fundamentales para la construcción de ciudadanos, porque sus contenidos revelan análisis y reflexiones acerca de las problemáticas sociales y políticas. La comprensión de las relaciones y los problemas que se viven en colectivo son materia de ciencias como la sociología, ciencia política y filosofía, por tanto, su intervención en la formación de ciudadanía es vital para que los jóvenes obtengan una consciencia crítica de su entorno y, por ende, de su participación. 
En este sentido, desde la visión de las ciencias exactas, se menciona la relación de la educación ciudadana a través del conocimiento científico como parte de un proceso cognitivo, porque hace referencia a los alcances de la ciencia en la sociedad y sus repercusiones en el medio ambiente. De modo que, la alfabetización científica es una alternativa para una mayor concientización en la toma de decisiones y en la sensibilidad social frente al desarrollo técnico-científico que puede generar riesgo al medio ambiente y a las personas.

Otra línea de la perspectiva de la educación en valores es la de Barba (2001,2005), Díaz Barriga (2005) y Barrios, 2014), quienes consideran a los valores como un proyecto que permite el razonamiento de los jóvenes acerca de su pertenencia a una sociedad, mediante la acción racional de llevar actos que promuevan la armonía social, por medio del compromiso y la responsabilidad.

Al hablar de las prácticas cotidianas y su vínculo con la formación ciudadana, aparece la perspectiva de Educación Social. Arias (2006) y Parcerisa (2008) mencionan que es una perspectiva integradora, que conduce a la resistencia y a la transformación al ser una educación integral, donde los estudiantes se relacionan con su contexto, donde se interviene lo público y lo privado. La formación integral busca que el individuo se relacione con la comunidad y también con el mundo productivo, científico, social, político y cultural.

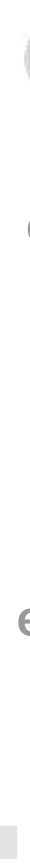

Ochman y Cantú (2013) lanzan una propuesta de evaluación de las ocho competencias que son parte del currículo dentro de los planes de estudio de la educación media superior. En la propuesta se evaluarán la perspectiva social y búsqueda del bienestar común, el respeto a la diversidad, el manejo y resolución de problemas, la participación democrática, respeto y valoración de la legalidad, la comprensión democrática como sistema de gobierno, la comprensión de la democracia como forma de vida, y el ejercicio democrático de la autoridad y control ciudadano del poder. En el artículo no se muestran resultados concretos, pero hace hincapié en que la formación ciudadana se vive en la vida escolar y cotidiana.

Las propuestas presentadas son sumamente interesantes porque, desde su perspectiva, invitan al 
En este mismo sentido, los jóvenes representan el motor de cambio en la forma de manifestarse por medio del uso de las nuevas herramientas tecnológicas. Menciona la Encuesta Iberoamericana sobre la Juventud que 51\% de los jóvenes tiene acceso a internet, en donde pasan el tiempo navegando en redes sociales e informándose acerca de temas de diversas índoles (2014). Con respecto a la cultura política de los jóvenes mexicanos se menciona, en la misma encuesta, que está muy por debajo de otros países al solamente tener 5\% de confianza en la política y $8 \%$ en el gobierno. Lo que hay que destacar es que aún encuentran esperanza en la democracia con 15\%.

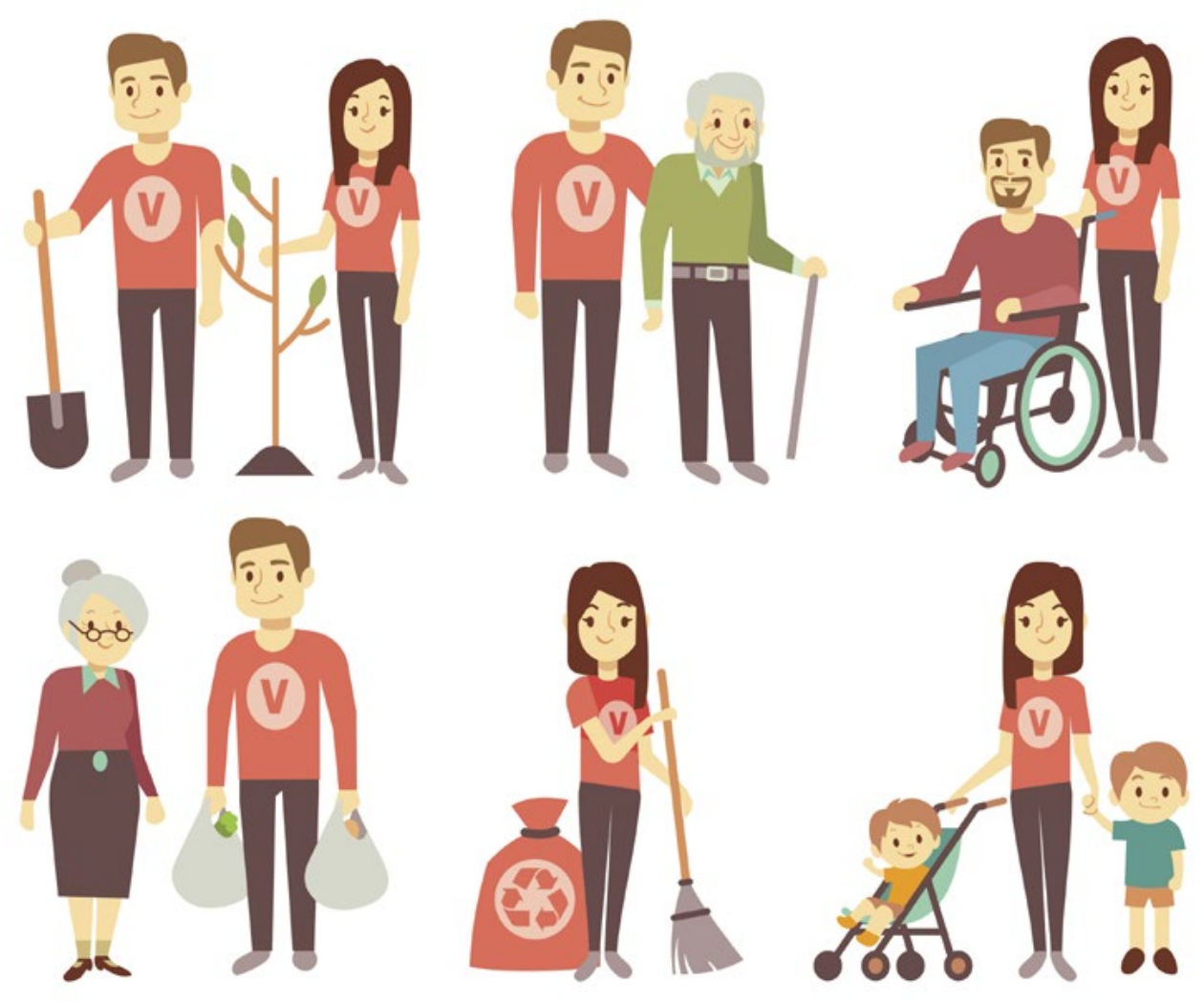

La falta de participación deriva en gran medida en la poca identificación con las instituciones y la resolución de las demandas por parte de los aparatos del Estado (partidos políticos, instituciones, representantes del poder). De igual forma, el poco interés por la información afecta en la participación para mejorar su entorno, de forma que repercute en una ciudadanía participativa (Bustelo, 1998).

Sin embargo, la participación ciudadana de los jóvenes tiene muchos matices, ellos han encontrado otras alternativas para organizarse y expresar su desacuerdo con lo establecido socialmente. El conflicto es uno de los principales motivos para iniciarse en la participación y en muchos de los casos se debió a un 
descontento individual, el cual tiene impacto en otros grupos de jóvenes, lo que puede convertirse en demandas sociales.

Henao et al. (2008), Peralta (2009) y Martínez et al. (2010) analizan la relaciones que establecen los jóvenes a partir de una inconformidad, las actitudes que toman y los compromisos ante la adversidad. La organización y la reivindicación de derechos para la protección del ambiente y la defensa de las culturas originarias, por medio de las redes sociales han sido el motor para crear organizaciones juveniles. En este sentido, Peralta (2009) añade la importancia de una vinculación entre la educación y el ámbito público, porque los aportes desde la educación serán más sólidos en la conformación de una cultura política. Mientras tanto, Martínez (2010) enfatiza la importancia de que los jóvenes estrechen relaciones sociales y actitudes políticas para tener un mayor compromiso con los demás.

El surgimiento de colectivos en pro de los derechos culturales, sirve para revalorizar los derechos de las mujeres, de la aceptación de la diversidad, medio ambiente, animales; y fungen un papel importante en la creación de los nuevos movimientos sociales. Algunos grupos de jóvenes y colectivos salen a las calles a denunciar las injusticias y la nueva valorización de las sociedades actuales.

Dentro de la cultura ciudadana el compromiso y la participación figura en los jóvenes como interés en los temas que se consideran emergentes. Por ejemplo, en el documental Comprar, tirar comprar, Dannoritzer (2011) muestra como un grupo de adolescentes se organiza y denuncia el poco rendimiento de las baterías Apple en los reproductores musicales; mediante una campaña de pintas en las paredes, se señalaba la escasa duración y lo obsoleto del producto después de un año de uso. De tal manera, se pone en duda la calidad y tecnología de Apple, que se jacta de su durabilidad y de ser amigable con el medio ambiente. Las protestas tuvieron impacto en las redes sociales, a tal grado que se llevó el caso a los juzgados, en donde se determinó que la compañía debía de reparar los daños a los consumidores, y se impulsó una nueva ley para prolongar la vida útil de las baterías y contar con un recambio de ella, para el beneficio del medio ambiente. La organización juvenil tuvo un impacto importante para crear un movimiento para el cuidado de la naturaleza y la denuncia a grandes compañías que operan mediante el engaño y en beneficio de sus propios intereses.

Otro ejemplo de participación ciudadana es mediante la denuncia, en este caso, la fotografía. Fernando Brito (2011) hace una crítica creativa acerca de lo que ocurre con el narcotráfico en el norte del país, expresa mediante su óptica una realidad cotidiana e impune de lo que sucede en la guerra entre bandas de narcotraficantes por la posesión del territorio. Su participación en varios concursos internacionales mostró la violencia y el desgajamiento de las instituciones de nuestro país.

Unos de los recursos más recurrentes para exponer las inconformidades, denuncias y propuestas para el mejoramiento del entorno son las redes sociales, este es el caso de un grupo de jóvenes denominados "Los supercívicos" 
(2013), quienes denuncian actos no ciudadanos en circunstancias cotidianas, como es el respeto de los espacios para las personas con discapacidad, el ceder un asiento a la mujer embarazada o al adulto mayor. Mediante una denuncia con toques de humor, se muestran las faltas, para las que en ocasiones no existe sanción, pero que sí quebrantan la convivencia. En este mismo sentido, existe otro grupo de jóvenes que realizan experimentos sociales, en los cuales se filma la honradez y responsabilidad, buscando concientizar, a partir de acciones, el deber ciudadano; como ellos mismos lo mencionan "buscar encontrar nuevamente la fe en la humanidad" (Mensajeros urbanos, 2013).

Por tanto, algunas propuestas de formación ciudadana se vinculan con la cultura ciudadana y coadyuvan con la participación de los jóvenes de bachillerato. Una de las alternativas actuales es el uso de los medios de comunicación como un factor importante para la construcción de ciudadanía (Palencia, 2005). El autor subraya el déficit por parte de la cultura cívica de las instituciones educativas, porque sus contenidos son aburridos, aislados del contexto social y solamente promueven la identidad nacional a través del culto de los símbolos patrios y ceremonias, desdeñando en el fondo al sistema político, sus prácticas y su impacto sociocultural, para olvidar su carácter formativo de la ciudadanía. La propuesta se centra en generar contenidos en las redes sociales, porque ahí se establecen espacios para la expresión libre de sentimientos, relaciones y corporalidades. La importancia de orientar el uso crítico de la información y la participación con los jóvenes permite una consciencia acerca de los temas políticos que acontecen y su contexto.

Begoña y Contreras (2006) promueven el uso de las Tecnologías de Información y Comunicación (TIc), mediante una vigilancia de contenidos y participación activa con las competencias ciudadanas para crear espacios públicos digitales. Se alude que la mayoría de las interacciones se basan en el consumo, pero que tam-

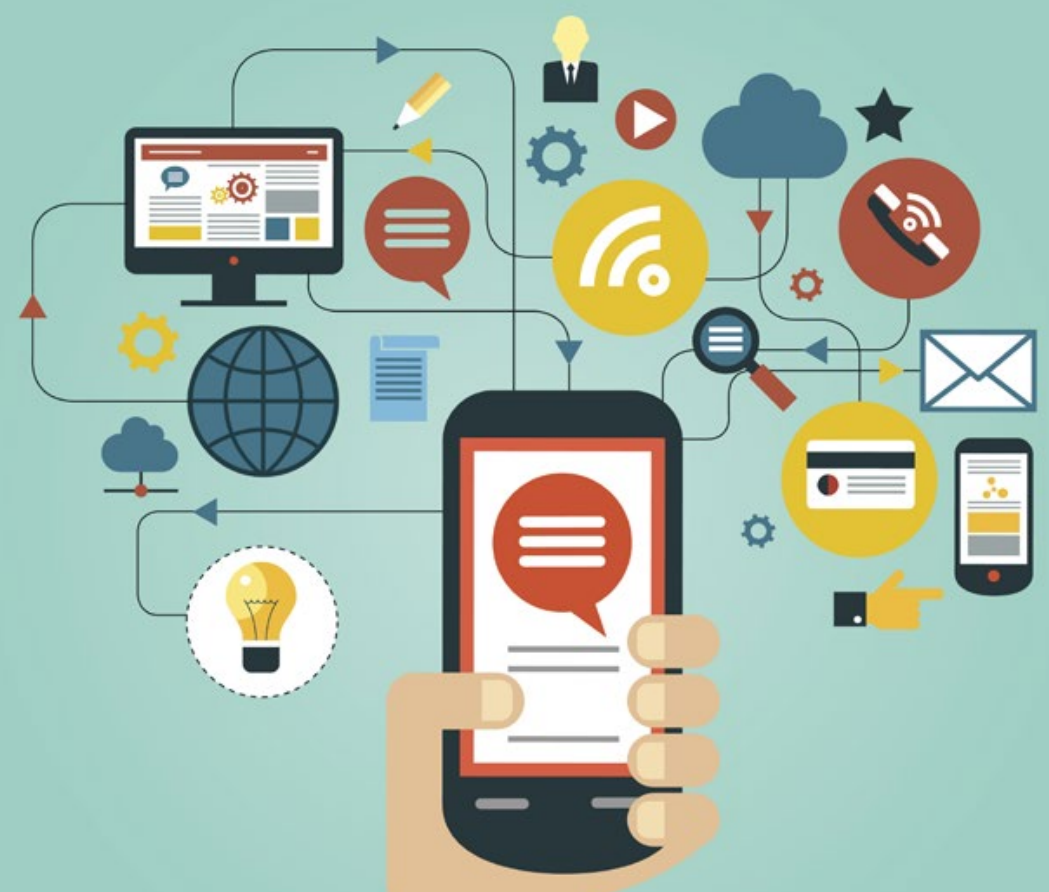
bién se crean constantemente foros de discusión, participación y reflexión acerca de temas contemporáneos. Ahí se reconocen los derechos y la solución a demandas actuales. Por tanto, se busca generar espacios, contenidos y acciones, en donde los jóvenes se expresen y participen en beneficio de ellos y su entorno.

En la actualidad se caracteriza a los jóvenes como propietarios del uso constante de las TIC, como nativos digitales, porque se les distingue por el intercambio constante de información, ideas, reflexiones, críticas y propuestas, en relación con lo que ocurre en su situación personal y de su entorno. En el caso de la participación política, los jóvenes se caracterizan por viralizar situaciones comprometedoras de funcionarios públicos 
o ciudadanos, y, en ocasiones, manifiestan su apoyo a campañas de cuidado del ambiente, defensa de los derechos animales y de los grupos en situación de discriminación. Otros más activos se pronuncian como veganos, anarquistas o pertenecientes a ideologías distintas a lo que proponen los partidos políticos. Sin embargo, la gran mayoría de los jóvenes se dedican a una participación pasiva, a solamente reetiquetar la información, opinar pero sin ninguna actuación, se encuentran más preocupados por el consumo y el hedonismo que por las cuestiones de su entorno. La propuesta de un uso adecuado de la información busca incurrir en la participación y en la reflexión crítica que proporciona la educación, mediante el desarrollo de las competencias ciudadanas para actuar en el contexto en donde se vive.

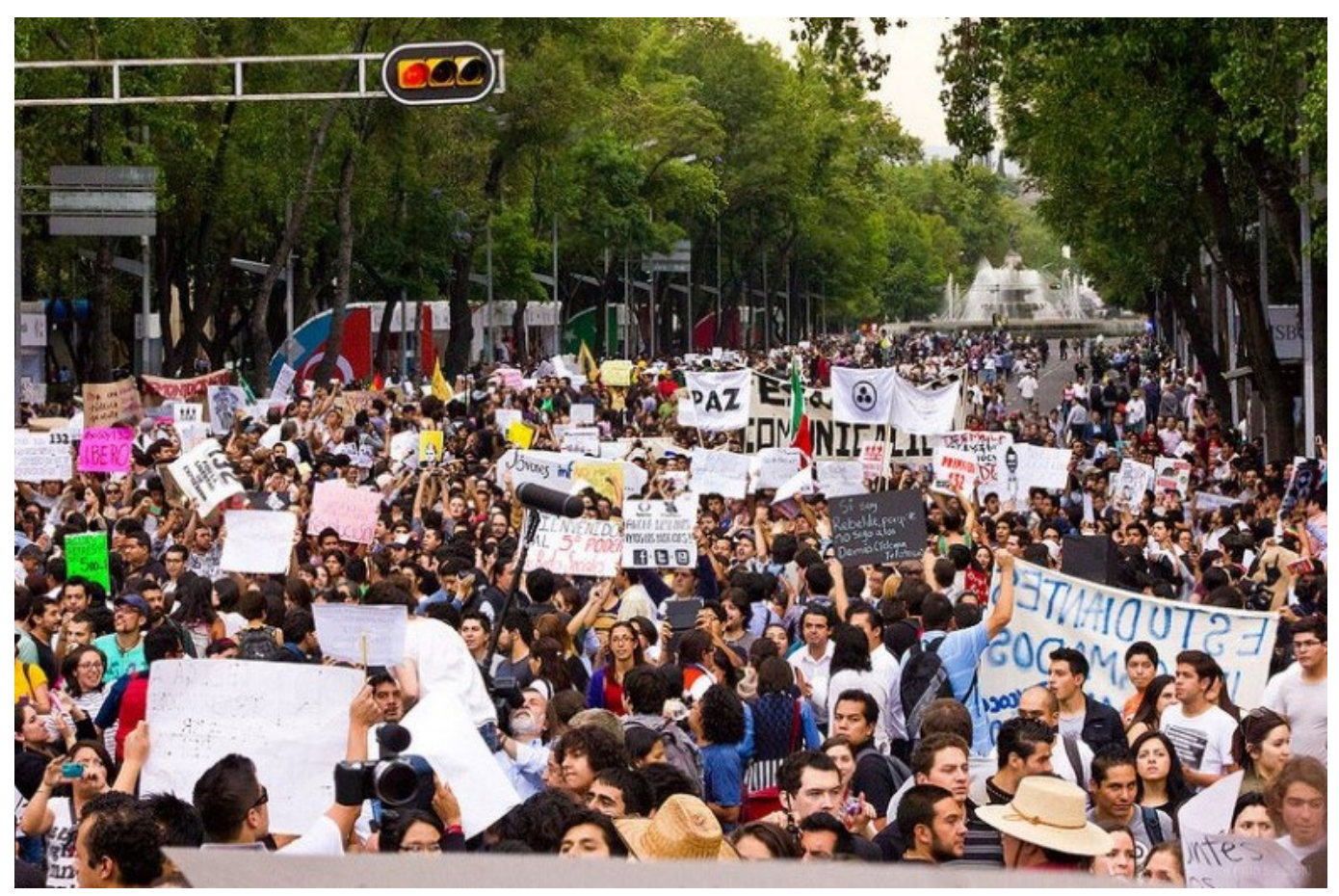

\section{A manera de cierre}

A lo largo del artículo se han observado diversas posturas con respecto a la formación ciudadana de los estudiantes de bachillerato, se han planteado alternativas desde los planes curriculares, transversalidad en contenidos (desde las ciencias naturales hasta las humanidades), mediante las TIC, reflexiones acerca de los valores, fomento hacia el trabajo colaborativo, hasta una educación social en donde se involucren a todos los actores de una sociedad para que se reconozca la diversidad.

La construcción de una cultura ciudadana en México es incipiente, al tener pocos elementos estructurales que permitan a los ciudadanos a participar de 
forma directa en las decisiones políticas del país. Sin embargo, los jóvenes han abierto nuevas formas de participación y actuación, a través de otros medios de expresión, dando lugar a ciudadanos preocupados por su entorno. Algunos ejemplos son: las manifestaciones por los derechos culturales de la diversidad sexual, género, pueblos indígenas, jóvenes y formas de expresión, así como la defensa de los planes de estudios de las universidades. Se han creado asociaciones colectivas por la defensa del medio ambiente, los derechos de los animales, apoyo a los niños de la calle, foros de expresión artística y cultura.

Asimismo, ha tomado énfasis el respeto a las normas sociales de convivencia: ciertos colectivos, por medio de las de las redes sociales y el ciberespacio, hacen denuncias y ponen en evidencia a los trasgresores, ya sean servidores públicos o los mismos ciudadanos (ceder el asiento en el transporte público, no tirar basura, el respeto de los espacios para personas con discapacidad, ciclistas, multas de tránsito, etcétera). También existen pequeños colectivos que reafirman sus condiciones individuales y permiten la expresión en diferentes formas, como son las artísticas o de apoyo a grupos en situación de vulnerabilidad.

La cultura ciudadana mexicana se encuentra viva, pero aún falta un mayor fomento y fortalecimiento de ella, y esto se logra mediante la interacción con diversas personas, el respeto y compromiso para participar en temas comunes, y mediante el apoyo mutuo para lograr cambios en la comunidad. Una formación ciudadana que vincule las dos facetas, la del compromiso por entregar contenidos y aprendizajes sobre la ciudadanía, así como una responsabilidad social por llevarlos a cabo en la vida cotidiana fomentará una cultura ciudadana dentro de las escuelas, donde se reconocerá al conflicto como un elemento circunstancial de la sociedad; $y$, en el reconocimiento de las diversas formas de participar activamente por los diferentes asuntos que conciernen a la vida pública, se ampliaría el abanico de posibilidades de injerencia de los futuros ciudadanos.

Se requiere que los estudiantes de bachillerato reconozcan el conflicto que vive su medio actual y que reflexionen de forma crítica a partir de la información que les proporcionen sus profesores y su mismo entorno; que se incite a la participación, a luchar por la libertad, al reconocimiento de la diversidad, a través de un ambiente que propicie la responsabilidad, el compromiso y el intercambio de ideas.

\section{Referencias}

* Alcántara, A. y Zorrilla (2006). Globalización y Educación Media Superior en México. En busca de la pertinencia curricular. Revista Perfiles educativos, 127, 38-57.

- Almond, G. y Bingham, P. (1972). Política comparada. Una concepción evolutiva. Buenos Aires: Paidós. 
* Arias, R. L. (2006). Educación Social y formación ciudadana en tiempos de globalización. Revista Tolerancia, 11, 117-129. Recuperado de: http://www.ts.ucr.ac.cr/ binarios/tendencias/rev-co-tendencias-11-08.pdf.

* Barba, B. (2005). Educación y valores. Una búsqueda para reconstruir la convivencia. Revista RMIE, 10(24), 9-14. Recuperado de: http://temoa.itesm.mx/es/ node/45917.

* Bolívar, A. y Belaguer, F. (2014). La educación para la ciudadanía: marco pedagógico y normativo. Recuperado de: http://studylib.es/doc/481328/la-educaciónpara-la-ciudadan\%C3\%ADa--marco-pedagógico-y-norma.

* Brito, F. (2011). Tus pasos se perdieron con el paisaje [fotografía]. Culiacán: xIv Bienal de fotografía México 2010 [premio descubrimiento FotoEspaña, 2011].

* Bustelo, E. y Milujin, A. (1998). La política social esquiva, en revista de Ciencias Sociales. Buenos Aires: Universidad Nacional de Quilmes.

- Carrillo, P. (2013). Relevancia de las humanidades y las ciencias sociales. Eutopia, 17, 98-100. Recuperado de: http://www.journals.unam.mx/index.php/eutopia/article/view/42234.

* Castro, M. I., Rodríguez, A. y Smith, M. (2014). La construcción de ciudadanía en la educación media superior. Un estudio de caso sobre docentes de la unam. México: IISUE.

* Dannoritzer, C. (2011). Comprar, tirar, comprar [documental]. Madrid: RTVE.

* Díaz Barriga, A. (2005). La educación en valores: Avatares del curriculum formal, oculto y los temas transversales. Redie, 7(2), 1-15. Recuperado de: http://redie. uabc.mx/redie/article/view/117.

- Flores, J. y Meyenberg, Y. (2000). Ciudadanos y cultura de la democracia. Reg/as, Instituciones y valores de la democracia. México: IFE.

* Gil Pérez, D. y Vilches, A. (2006).Educación ciudadana y alfabetización científica: mitos y realidades. Revista Iberoamericana en educación, 42, 31-53. Recuperado de: http://rieoei.org/rie42a02.htm.

* Gros, B. y Contreras, D. (2006). La alfabetización digital y el desarrollo de competencias ciudadanas. Revista Iberoamericana de Educación, 42, 103-125. Recuperado de: http://www.oei.es/historico/noticias/spip.php?article582.

* Henao-Escovar, J., Ocampo-Talero, A. M., Robledo-Gómez, A. M. y Lozano-Ardila, M. C. (2008). Los grupos juveniles universitarios y la formación ciudadana. Revista Pontificia Universidad Javeriana, 7(3), 853-867. Recuperado de: http://www.scielo. org.co/pdf/rups/v7n3/v7n3a18.pdf.

* Instituto Mexicano de la Juventud (Imjuve). (2011). Encuesta Nacional de Juventud 2010, Resultados generales. México: IMJ.

* Instituto Nacional para la Evaluación de la Educación. (2015). Panorama educativo de México 2014. Indicadores del Sistema Educativo Nacional, educación básica y media superior. México: INEE. 
* Krotz, E. y Winocur, R. (2007). Democracia, participación y cultura ciudadana: discursos normativos homogéneos versus prácticas y representaciones heterogéneas. Revista Estudios sociológicos, xxv(73), 187-218. Recuperado de: http://www. redalyc.org/articulo.oa?id=59807307.

- Los supercívicos. (s. f.). Home [canal de YouTube]. Recuperado de: https://www. youtube.com/user/HoustonPrograma/featured.

- Mensajero urbanos. (s. f.). Home [canal de YouTube]. Recuperado de: https://www. youtube.com/channel/UCMz8xd5dLUUXG4I5W4AOt0A.

* Organización para la Cooperación y el Desarrollo Económicos. (2016). Programa para la Evaluación Internacional de alumnos (PISA) 2015- Resultados (1-14) México: oCDE. Recuperado de: http://www.oecd.org/pisa/pisa-2015-results-in-focus-ESP. pdf.

* Pagés, J. (2003). Ciudadanía y enseñanza de la historia. Reseñas de Enseñanza de la Historia, 1, 11-42. Recuperado de: https://www.researchgate.net/publication/228768216 Ciudadania y ensenanza de la historia.

* Palencia Villa, M. (2005). La formación ciudadana de los jóvenes a través de las redes sociales: Discusión sobre el capital social. Revista Espacio Abierto, 14(1), 97-117. Recuperado de: http://www.bibliotecadigital.uchile.cl/client/es ES/sisib/search/detailnonmodal/ent:\$002f\$002fSD ILS\$002f0\$002fSD ILS:504985/ ada?qu=Mercados+--+Investigaciones.\&qf=SUBJECT\%09Materia\%09Capital+soc ial+--+México.\%09Capital+social+--+México.\&ic=true\&ps=1000.

- Parcerisa Aran, A. (2008). Educación Social en y con la institución escolar. Revista interuniversitaria de Pedagogía Social, 15, 15-27. Recuperado de: https://www.academia.edu/15366996/Educación Social en y con la institución escolar.

* Peralta, B. (2009). La formación ciudadana en el sistema educativo de Colombia: ¿Una mirada reactiva o transformadora? Revista Eleuthera, Bogotá. 165-178. Recuperado de: http://eleuthera.ucaldas.edu.co/downloads/Eleuthera3 6.pdf. consultado el 16 de mayo de 2017.

* Peschard, J. (1996). La cultura política democrática, transición de fin de sig/o. México: Instituto Federal Electoral.

* Secretaria de Educación Pública. (2008). Proyecto de Reforma Integral de la Educación Media Superior en México. La creación de un sistema nacional de bachillerato en un marco de la diversidad. México: SEP.

* Secretaria de Educación Pública. (2012). Formación ciudadana, plan de estudios ems (1-22). México: SEP. http://www.dgespe.sep.gob.mx/public/rc/programas/lepree/ formacion ciudadana lepree.pdf.

- Secretaría de Gobernación. (2013). Encuesta Nacional sobre cultura política y prácticas ciudadanas 2012. México: Secretaria de Gobernación.

* Universidad Nacional Autónoma de México. (2012). Documento base para la actualización del plan de estudios: doce puntos a considerar. México: unam. Recuperado de: http://www.cch.unam.mx/sites/default/files/Docuemento base.pdf. 


\section{Cómo CitAR ESTe ARTículo}

* García Reyes, Jesús (2018). La formación ciudadana en estudiantes de bachillerato. Revista Digital Universitaria (RDU). Vol. 19, núm. 5 septiembre-octubre. DOI: http://doi.org/10.22201/codeic.16076079e.2018.v19n5.a7. 